\title{
Strong Approximation of Empirical Copula Processes by Gaussian Processes
}

\author{
Salim BOUZEBDA* and Tarek ZARI ${ }^{\dagger}$ \\ L.S.T.A., Université Pierre et Marie Curie \\ 4 place Jussieu 75252 Paris Cedex 05, France
}

October 31, 2018

\begin{abstract}
We provide the strong approximation of normalized empirical copula process by a Gaussian process. In addition we establish a strong approximation of the smoothed empirical copula processes and a law of iterated logarithm.
\end{abstract}

AMS Subject Classifications: Primary 60F17 ; secondary 62G20 ; 62H10 ; 60F15.

Keywords: Empirical Copula processes ; Strong invariance principles ; Kernel-type-estimator ;

Kiefer processes ; Gaussian processes.

\section{Introduction}

The aim of the present paper is to provide the strong approximations of the normalized empirical copula process $\left\{\mathbb{A}_{n}(\mathbf{u}): \mathbf{u} \in[0,1]^{d}, \quad n \geq 1\right\}$, (see, e.g., (1.10) below for definition), by a single Gaussian process $\left\{\mathscr{K}_{\mathbb{C}}^{*}(\mathbf{u}, n): \mathbf{u} \in[0,1]^{d}, \quad n \geq 1\right\}$. Thus, we get the strong approximations of $\left\{\mathbb{A}_{n}(\mathbf{u}): \mathbf{u} \in\right.$ $\left.[0,1]^{d}, \quad n \geq 1\right\}$ in terms of Gaussian process in both $\mathbf{u}$ and $n$. We will mainly be concerned with the general case, in which $\left\{\mathbb{A}_{n}(\mathbf{u}): \mathbf{u} \in[0,1]^{d}, n \geq 1\right\}$ is generated by a sample of random vectors with dependent marginals.

Consider a continuous random vector $\mathbf{X}=\left(X_{1}, \ldots, X_{d}\right)$ with joint cumulative distribution function $\mathbb{F}(\mathbf{x})=\mathbb{P}(\mathbf{X} \leq \mathbf{x})=\mathbb{P}\left(X_{1} \leq x_{1}, \ldots, X_{d} \leq x_{d}\right)$, for $\mathbf{x} \in \mathbb{R}^{d}$, and margins $F_{1}(\cdot), \ldots, F_{d}(\cdot)$. Here and elsewhere, for $\mathbf{x}=\left(x_{1}, \ldots, x_{d}\right)$ and $\mathbf{y}=\left(y_{1}, \ldots, y_{d}\right)$, we write $\mathbf{x} \leq \mathbf{y}$ to denote that $x_{j} \leq y_{j}$, for $j=1, \ldots, d$. The characterization theorem of Sklar (1959) implies that there exists a copula function $\mathbb{C}(\cdot)$ on $[0,1]^{d}$, such that

$$
\mathbb{F}(\mathbf{x})=\mathbb{C}\left(F_{1}\left(x_{1}\right), \ldots, F_{d}\left(x_{d}\right)\right), \text { for } \mathbf{x}=\left(x_{1}, \ldots, x_{d}\right) \in \mathbb{R}^{d} .
$$

This copula, which is unique, is a multivariate cumulative distribution function whose univariate marginals are uniform on the interval $(0,1)$. The Sklar's theorem provides the theoretical foundation for the

*e-mail: salim.bouzebda@upmc.fr (Corresponding author)

†e-mail: zaritarek@gmail.com 
widespread use of the copula approach in generating multivariate distributions from univariate distributions. The copula function pertaining to $\mathbb{F}(\cdot)$ may be defined by

$$
\mathbb{C}(\mathbf{u})=\mathbb{F}\left(F_{1}^{-}\left(u_{1}\right), \ldots, F_{d}^{-}\left(u_{d}\right)\right) \text { for } \mathbf{u}=\left(u_{1}, \ldots, u_{d}\right) \in[0,1]^{d},
$$

where, for $j=1, \ldots, d, F_{j}^{-}(u)=\inf \left\{x \in \mathbb{R}: F_{j}(x) \geq u\right\}$, with $u \in[0,1]$, denotes the quantile function of $F_{j}(\cdot)$. In the monographs by Nelsen (2006) and Joe (1997) the reader may find detailed ingredients of the modelling theory as well as surveys of the commonly used copulas. For in depth and overview historical notes we refer to Schweizer (1991). Copulas have proved to be a flexible and versatile tool in the analysis of dependency structures. To be more specific, copula $\mathbb{C}(\cdot)$ "couples" the joint distribution function $\mathbb{F}(\cdot)$ to its univariate marginals, capturing as such the dependence structure between the components of $\mathbf{X}=\left(X_{1}, \ldots, X_{d}\right)$. Indeed, most conventional measures of dependence can be explicitly expressed in terms of the copula. This feature has motivated successful applications in actuarial science and survival analysis (see, e.g., Frees and Valdez (1998), Cui and Sun (2004)). In the literature on risk management and, more generally, in mathematical economics and mathematical finance modelling, a number of illustrations are provided (refer to books of Cherubini et al. (2004) and McNeil et al. (2005)), in particular, in the context of asset pricing and credit risk management. First, we shall introduce some notations and definitions which will be used for the statement of our forthcoming results. Let $\mathbf{X}_{i}=\left(X_{1 i}, \ldots, X_{d i}\right), i=1,2, \ldots$, be independent random vectors with common distribution function $\mathbb{F}(\cdot)$ whose margins $F_{1}(\cdot), \ldots, F_{d}(\cdot)$ are continuous and whose copula is denoted by $\mathbb{C}(\cdot)$. Define $U_{j i}=F_{j}\left(X_{j i}\right)$, for $i=1, \ldots, n$ and $j=1, \ldots, d$. The random vectors $\mathbf{U}_{i}=\left(\xi_{1 i}, \ldots, \xi_{d i}\right)$ constitute an i.i.d. sample from $\mathbb{C}(\cdot)$. Setting $\mathbb{1}\{\cdots\}$ for the indicator function of $\{\cdots\}$, we define, for each $n \geq 1$, the following empirical distribution functions, for $\mathbf{x} \in \mathbb{R}^{d}$ and for $\mathbf{u} \in[0,1]^{d}$,

$$
\begin{aligned}
\mathbb{F}_{n}(\mathbf{x}) & :=\frac{1}{n} \sum_{i=1}^{n} \prod_{j=1}^{d} \mathbb{1}\left\{X_{j i} \leq x_{j}\right\}, \\
F_{j n}\left(x_{j}\right) & :=\frac{1}{n} \sum_{i=1}^{n} \mathbb{1}\left\{X_{j i} \leq x_{j}\right\}, \\
\mathbb{G}_{n}(\mathbf{u}) & :=\frac{1}{n} \sum_{i=1}^{n} \prod_{j=1}^{d} \mathbb{1}\left\{U_{j i} \leq u_{j}\right\}, \\
G_{j n}\left(u_{j}\right) & :=\frac{1}{n} \sum_{i=1}^{n} \mathbb{1}\left\{U_{j i} \leq u_{j}\right\} .
\end{aligned}
$$

The marginal quantile functions associated to $F_{j n}(\cdot)$ and $G_{j n}(\cdot)$, for $j=1, \ldots, d$ and $u_{j} \in[0,1]$, are defined by

$$
\begin{aligned}
F_{j n}^{-}\left(u_{j}\right) & :=\inf \left\{x \in \mathbb{R}: F_{j n}(x) \geq u_{j}\right\}, \\
G_{j n}^{-}\left(u_{j}\right) & :=\inf \left\{u \in[0,1]: G_{j n}(u) \geq u_{j}\right\} .
\end{aligned}
$$

According to Deheuvels (1979), in view of the characterization (1.1), we define an empirical copula function of $\mathbb{F}_{n}(\cdot)$, based upon $\mathbf{X}_{1} \ldots \mathbf{X}_{n}$, as any copulas $\mathbb{C}_{n}(\cdot)$ fulfilling the fundamental identity

$$
\mathbb{C}_{n}(\mathbf{u}):=\mathbb{F}_{n}\left(F_{1 n}^{-}\left(u_{1}\right), \ldots, F_{j n}^{-}\left(u_{d}\right)\right), \text { for } \mathbf{u} \in[0,1]^{d} .
$$


It may be assumed without loss of generality that the marginal distributions of $\mathbb{F}(\cdot)$ are uniform on the interval $[0,1]$, or equivalently that $\mathbb{F}(\cdot)$ is a copula, i.e., we can work, in the sequel, directly with the sample $\mathbf{U}_{1}, \ldots, \mathbf{U}_{n}$ from $\mathbb{C}(\cdot)$. Here, we may refer to Sklar $(1959,1973)$, Philipp and Pinzur (1980), Wichura (1973) and Moore and Spruill (1975) among others. It follows that the empirical copula in equation (1.8) is given by

$$
\mathbb{C}_{n}(\mathbf{u}):=\mathbb{G}_{n}\left(G_{1 n}^{-}\left(u_{1}\right), \ldots, G_{j n}^{-}\left(u_{d}\right)\right), \text { for } \mathbf{u} \in[0,1]^{d} .
$$

The empirical copula function $\mathbb{C}_{n}(\cdot)$ was briefly discussed by Ruymgaart (1973), pp. 6-13, in the introduction of his doctoral thesis. The asymptotic behavior of $\mathbb{C}_{n}(\cdot)$ was studied in several papers, including Deheuvels (1979), Stute (1984), Gaenssler and Stute (1987), Rüschendorf (1974, 1976) or Tsukahara (2005) and the references therein. We may now define the normalized empirical copula process $\mathbb{A}_{n}(\cdot)$, according to Rüschendorf (2009), by setting

$$
\mathbb{A}_{n}(\mathbf{u}):=n^{1 / 2}\left(\mathbb{C}_{n}(\mathbf{u})-\mathbb{C}(\mathbf{u})\right), \quad \text { for } \quad \mathbf{u} \in[0,1]^{d} .
$$

The asymptotic behavior of the process $\left\{\mathbb{A}_{n}(\mathbf{u}): \mathbf{u} \in[0,1]^{d} ; n>0\right\}$ has been investigated extensively in stochastic literature. Deheuvels $(1980,1981)$ obtained the exact law and the limiting process of $\left\{\mathbb{A}_{n}(\mathbf{u}): \mathbf{u} \in[0,1]^{d} ; n>0\right\}$ under independence assumption of margins. Rüschendorf $(1974,1976)$ and Gaenssler and Stute (1987) proved weak convergence of the process $\left\{\mathbb{A}_{n}(\mathbf{u}): \mathbf{u} \in[0,1]^{d} ; n>0\right\}$ in the space $D\left([0,1]^{2}\right)$, where the space of càdlàg functions $D\left([0,1]^{2}\right)$ equipped with the Skorohod topology. van der Vaart and Wellner (1996) established weak convergence in the space $\ell^{\infty}\left([a, b]^{2}\right)$, when $0<a<b<1$, under restrictions on the distribution functions. Fermanian et al. (2004) showed that the weak convergence of $\mathbb{A}_{n}(\cdot)$ to a centered Gaussian process holds on $\ell^{\infty}\left([0,1]^{2}\right)$, when $\mathbb{C}(\cdot)$ has continuous partial derivatives on $[0,1]^{2}$. Recently, Segers (2010) showed that the weak convergence of the normalized empirical copula process under the assumption that the first-order partial derivatives of the copula exist and are continuous on certain subsets of the unit hyper-cube. We can say that the strong approximation holds for the process $\left\{\mathbb{A}_{n}(\mathbf{u}): \mathbf{u} \in[0,1]^{d} ; n>0\right\}$ with rate $\left(b_{n}\right)$, this means that, on a suitable probability space $(\Omega, \mathscr{A}, \mathbb{P})$,

$$
\sup _{\mathbf{u} \in[0,1]^{d}}\left|\mathbb{A}_{n}(\mathbf{u})-\mathbb{Z}_{n}(\mathbf{u})\right|=O\left(b_{n}\right), \text { a.s. },
$$

where $\mathbb{Z}_{n}(\cdot)$ is a sequence of Gaussian processes and $b_{n} \rightarrow 0$ is a deterministic rate. The strong approximations are quite useful and have received considerable attention in probability theory. Indeed, many well-known probability theorems can be considered as consequences of results about strong approximation of sequences of sums by corresponding Gaussian sequences. We shall mention that the rates of convergence for the distribution of smooth functionals of $\left\{\mathbb{A}_{n}(\mathbf{u}): \mathbf{u} \in[0,1]^{d} ; n>0\right\}$ can also be deduced from the approximation in (1.11). The approximation by Kiefer processes is of particular interest, since any kind of law of the iterated logarithm which holds for the partial sums of Gaussian processes may then be transferred to the empirical processes $\left\{\mathbb{A}_{n}(\mathbf{u}): \mathbf{u} \in[0,1]^{d} ; n>0\right\}$. We refer to Komlós et al. (1975), (DasGupta, 2008, Chapter 12), (Csörgő and Horváth, 1993, Chapter 3), (Csörgố and Révész, 1981, Chapters 4-5) and (Shorack and Wellner, 1986, Chapter 12) for expositions and references about this problem. We refer to Csörgő and Hall (1984) for a survey of some applications of the strong approximation and many references. The interested reader is referred to Deheuvels (2009) and the references 
therein concerning the strong approximations for the process $\left\{\mathbb{A}_{n}(\mathbf{u}): \mathbf{u} \in[0,1]^{d} ; n>0\right\}$. In the last reference, a full characterization of empirical copula in general framework is provided. There is a huge literature on the strong approximations and their applications. It is not the purpose of this paper to survey this extensive literature.

The remainder of the present paper is organized as follows. In section 2.1, we will introduce notations and definitions regarding some Gaussian processes, which play a central role in the strong approximations theory. In section 2.2, we will give our main result concerning the strong approximations of empirical copula processes by a single Gaussian process, which is stated in Theorem 2.1 below. In section 3, we will give some applications of Theorem 2.1. More precisely, we transfer our result to smoothed version of $\left\{\mathbb{A}_{n}(\mathbf{u}): \mathbf{u} \in[0,1]^{d} ; n>0\right\}$ as well as to the law of the iterated logarithm for the normalized empirical copula process. To avoid interrupting the flow of the presentation, all mathematical developments are postponed to Section 4.

\section{Main results}

\subsection{Gaussian Processes}

Let $\mathbb{C}(\cdot)$ be any copula. The $d$-variate Wiener process $\left\{\mathbb{W}_{\mathbb{C}}(\mathbf{u}): \mathbf{u} \in[0,1]^{d}\right\}$ on the unit cube of $\mathbb{R}^{d}$ associated with the copula function $\mathbb{C}(\cdot)$, i.e., $\mathbb{W}_{\mathbb{C}}(\cdot)$ is a $d$-variate Gaussian process on $[0,1]^{d}$ with

$$
\begin{gathered}
\mathbb{E}\left(\mathbb{W}_{\mathbb{C}}(\mathbf{u})\right)=0 \\
\mathbb{E}\left(\mathbb{W}_{\mathbb{C}}(\mathbf{u}) \mathbb{W}_{\mathbb{C}}(\mathbf{v})\right)=\mathbb{C}(\mathbf{u} \wedge \mathbf{v})
\end{gathered}
$$

where $\mathbf{u} \wedge \mathbf{v}:=\left(u_{1} \wedge v_{1}, \ldots, u_{d} \wedge v_{d}\right)$ for $\mathbf{u}=\left(u_{1}, \ldots, u_{d}\right) \in[0,1]^{d}$ and $\mathbf{v}=\left(v_{1}, \ldots, v_{d}\right) \in[0,1]^{d}$, and $\mathbb{W}_{\mathbb{C}}\left(u_{1}, \ldots, u_{d}\right)=0$ whenever $u_{j}=0, j=1, \ldots, d$.

A $d$-variate Brownian bridge process on $[0,1]^{d}$ associated with the copula function $\mathbb{C}(\cdot)$ is defined, in terms of $\mathbb{W}_{\mathbb{C}}(\cdot)$, by setting

$$
\mathbf{B}_{\mathbb{C}}(\mathbf{u}):=\mathbb{W}_{\mathbb{C}}(\mathbf{u})-\mathbb{C}(\mathbf{u}) \mathbb{W}_{\mathbb{C}}(\mathbf{1}), \text { for } \mathbf{u} \in[0,1]^{d}
$$

where $1:=(1, \ldots, 1)$. This process has continuous sample paths and fulfills

$$
\begin{aligned}
\mathbb{E}\left(\mathbf{B}_{\mathbb{C}}(\mathbf{u})\right) & =0 \\
\mathbb{E}\left(\mathbf{B}_{\mathbb{C}}(\mathbf{u}) \mathbf{B}_{\mathbb{C}}(\mathbf{v})\right) & =\mathbb{C}(\mathbf{u} \wedge \mathbf{v})-\mathbb{C}(\mathbf{u}) \mathbb{C}(\mathbf{v}), \text { for } \mathbf{u}, \mathbf{v} \in[0,1]^{d} .
\end{aligned}
$$

The interested reader may refer to Piterbarg (1996) and Adler (1990) for details on the Gaussian processes mentioned above. To state our result we need to define the Kiefer random field. Consider a $(d+1)$-variate Gaussian process $\mathbb{W}_{\mathbb{C}}(\mathbf{u}, z)$ on $[0,1]^{d} \times[0, \infty)$ such that $\mathbb{W}_{\mathbb{C}}(\mathbf{u}, z)=0$ whenever any of $u_{1}, \ldots, u_{d}$ or $z$ is zero. This process has continuous sample paths and fulfills

$$
\begin{aligned}
\mathbb{E}\left(\mathbb{W}_{\mathbb{C}}(\mathbf{u}, z)\right) & =0 \\
\mathbb{E}\left(\mathbb{W}_{\mathbb{C}}(\mathbf{u}, z) \mathbb{W}_{\mathbb{C}}(\mathbf{v}, t)\right) & =\min (z, t) \mathbb{C}(\mathbf{u} \wedge \mathbf{v})
\end{aligned}
$$


A $(d+1)$-variate Kiefer process $\mathscr{K}_{\mathbb{C}}(\cdot, \cdot)$ on $[0,1]^{d} \times[0, \infty)$ associated with the copula function $\mathbb{C}(\cdot)$, is defined, in term of $\mathbb{W}_{\mathbb{C}}(\cdot, \cdot)$, by setting

$$
\mathscr{K}_{\mathbb{C}}(\mathbf{u}, t):=\mathbb{W}_{\mathbb{C}}(\mathbf{u}, t)-\mathbb{C}(\mathbf{u}) \mathbb{W}_{\mathbb{C}}(\mathbf{1}, t)
$$

and fulfills

$$
\begin{aligned}
\mathbb{E}\left(\mathscr{K}_{\mathbb{C}}(\mathbf{u}, z)\right) & =0 \\
\mathbb{E}\left(\mathscr{K}_{\mathbb{C}}(\mathbf{u}, z) \mathscr{K}_{\mathbb{C}}(\mathbf{v}, t)\right) & =(z \wedge t)\{\mathbb{C}(\mathbf{u} \wedge \mathbf{v})-\mathbb{C}(\mathbf{u}) \mathbb{C}(\mathbf{v})\}
\end{aligned}
$$

for $\mathbf{u}, \mathbf{v} \in[0,1]^{d}$ and $z, t \geq 0$. We recall the distributional identity, for all fixed $z \geq 0$,

$$
z^{-1 / 2} \mathscr{K}_{\mathbb{C}}(\mathbf{u}, z) \stackrel{\mathscr{D}}{=} \mathbf{B}_{\mathbb{C}}(\mathbf{u})
$$

For each $n>0, u_{j} \in[0,1]$ and $j=1, \ldots, d$, the copula Gaussian process is defined by

$$
\begin{aligned}
\mathscr{K}_{\mathbb{C}}^{*}(\mathbf{u}, n) & :=\mathscr{K}_{\mathbb{C}}(\mathbf{u}, n)-\sum_{j=1}^{d} \mathscr{K}_{\mathbb{C}}\left(1, \ldots, 1, u_{j}, 1, \ldots, 1, n\right) \frac{\partial \mathbb{C}(\mathbf{u})}{\partial u_{j}} \\
& =: \quad \mathscr{K}_{\mathbb{C}}(\mathbf{u}, n)-\sum_{j=1}^{d} \mathscr{K}_{\mathbb{C}}^{(j)}\left(\mathbf{1}, u_{j}, \mathbf{1}, n\right) \frac{\partial \mathbb{C}(\mathbf{u})}{\partial u_{j}} .
\end{aligned}
$$

We are now in position to state our main results.

\subsection{Strong approximation results}

In the sequel, the precise meaning of "suitable probability space" is that an independent sequence of Wiener processes, which is independent of the originally given sequence of i.i.d. r.v., can be constructed on the assumed probability space. This is a technical requirement which allows for the construction of the Gaussian processes in our theorems, and is not restrictive since one can expand the probability space to make it rich enough (see e.g., Appendix 2 in Csörgő and Horváth (1993)).

The main result to be proved here may now be stated precisely as follows.

Theorem 2.1 Assume that $\mathbb{C}(\cdot)$, associated with $\mathbb{F}(\cdot)$, is twice continuously differentiable on $(0,1)^{d}$ and all the partial derivatives of second order are continuous on $[0,1]^{d}$. On a suitable probability space, we may define the empirical copula processes $\left\{\mathbb{A}_{n}(\mathbf{u}): \mathbf{u} \in[0,1]^{d}, n \geq 1\right\}$ in combination with the Gaussian process $\left\{\mathscr{K}_{\mathbb{C}}^{*}(\mathbf{u}, t): \mathbf{u} \in[0,1]^{d}, t \geq 0\right\}$, in such a way that, almost surely as $n \rightarrow \infty$

$$
\sup _{\mathbf{u} \in[0,1]^{d}}\left|\sqrt{n} \mathbb{A}_{n}(\mathbf{u})-\mathscr{K}_{\mathbb{C}}^{*}(\mathbf{u}, n)\right|=O\left(n^{1 / 2-1 /(4 d)}(\log n)^{3 / 2}\right)
$$

where $\mathscr{K}_{\mathbb{C}}^{*}(\mathbf{u}, t)$ is defined in (2.3).

The proof of Theorem 2.1 is captured in the forthcoming Section 4. 
Remark 2.2 In the particular case of independence, i.e.,

$$
\mathbb{C}(\mathbf{u})=\prod_{j=1}^{d} u_{j}
$$

the process $\left\{\mathscr{K}_{\mathbb{C}}^{*}(\mathbf{u}, n): \mathbf{u} \in[0,1]^{d} ; n \geq 0\right\}$ is equal to

$$
\mathscr{K}_{\mathbb{C}}^{*}(\mathbf{u}, n)=\mathscr{K}_{\mathbb{C}}(\mathbf{u}, n)-\sum_{j=1}^{d} \mathscr{K}_{\mathbb{C}}^{(j)}\left(\mathbf{1}, u_{j}, \mathbf{1}, n\right) \prod_{i \neq j}^{d} u_{i}, \quad \mathbf{u} \in[0,1]^{d},
$$

with mean zero and covariance function

$$
\mathbb{E}\left(\mathscr{K}_{\mathbb{C}}^{*}(\mathbf{u}, s) \mathscr{K}_{\mathbb{C}}^{*}(\mathbf{v}, t)\right)=(s \wedge t)\left\{\prod_{j=1}^{d}\left(u_{j} \wedge v_{j}\right)+(d-1) \prod_{j=1}^{d} u_{j} v_{j}-\sum_{j=1}^{d}\left(u_{j} \wedge v_{j}\right) \prod_{i \neq j} u_{i} v_{j}\right\},
$$

where $\mathbf{u}, \mathbf{v} \in[0,1]^{d}$ and $s, t \geq 0$. For more details the reader may refer to Csörgó (1979). Note that in the case where $\left\{\mathbb{A}_{n}(\mathbf{u}): \mathbf{u} \in[0,1]^{d} ; n>0\right\}$ is generated by a sample of random vectors with independent marginals then the limit distribution in (2.4) is free.

Remark 2.3 Theorem 2.1 may be used to derive the limiting laws of some statistics like Kendall's sample rank correlation coefficient and Spearman's sample rank correlation coefficient. More generally, let us define, for any function $J(\cdot)$ on $[0,1]^{3}$

$$
\mathbb{S}(\mathbb{C}):=\int_{0}^{1} \int_{0}^{1} J(u, v, \mathbb{C}(u, v)) d u d v .
$$

The corresponding sample quantity $\mathbb{S}\left(\mathbb{C}_{n}\right)$ may be called Spearman type rank statistic, the interested reader may refer to Gaenssler and Stute (1987) and Tsukahara (2000) for more details. To be more precise, suppose that $z \rightarrow J(u, v, z)$ has a continuous derivative $J^{3}(u, v, z)$ with $\sup _{u, v, z}\left|J^{3}(u, v, z)\right|=$ $\sup _{u, v, z}|\partial J(u, v, z) / \partial z|<\infty$. Then we can write

$$
\begin{aligned}
& \sqrt{n}\left(\mathbb{S}\left(\mathbb{C}_{n}\right)-\mathbb{S}(\mathbb{C})\right) \\
& =\sqrt{n}\left(\int_{0}^{1} \int_{0}^{1} J\left(u, v, \mathbb{C}_{n}(u, v)\right) d u d v-\int_{0}^{1} \int_{0}^{1} J(u, v, \mathbb{C}(u, v)) d u d v\right) \\
& =\int_{0}^{1} \int_{0}^{1} J^{3}\left(u, v, \delta_{n}(u, v)\right) \mathbb{A}_{n}(u, v) d u d v,
\end{aligned}
$$

where $\delta_{n}(u, v)$ is a point between $\mathbb{C}_{n}(u, v)$ and $\mathbb{C}(u, v)$, so that $\delta_{n}$ converge to $\mathbb{C}$ uniformly with probability one. Making use of Theorem 2.1 we have

$$
\left|\sqrt{n}\left(\mathbb{S}\left(\mathbb{C}_{n}\right)-\mathbb{S}(\mathbb{C})\right)-\int_{0}^{1} \int_{0}^{1} J^{3}(u, v, \mathbb{C}(u, v)) \frac{1}{\sqrt{n}} \mathscr{K}_{\mathbb{C}}^{*}(u, v, n) d u d v\right|=o_{\mathbb{P}}(1) .
$$

We put, for any function $J(\cdot)$ on $[0,1]^{3}$,

$$
\mathbb{T}(\mathbb{C}):=\int_{0}^{1} \int_{0}^{1} J(u, v, \mathbb{C}(u, v)) d \mathbb{C}(u, v),
$$

the integration being understood as multiple integral based on the bivariate copula. We call $\mathbb{T}\left(\mathbb{C}_{n}\right) a$ Kendall type rank statistic. Similarly, using Theorem 2.1 we can obtain the limiting law of $\sqrt{n}\left(\mathbb{T}\left(\mathbb{C}_{n}\right)-\right.$ $\mathbb{T}(\mathbb{C})$ ). 
Remark 2.4 Theorem 2.1 may be used to derive the limiting law of some smooth functionals. We can see this, in particular, for the Kolmogorov-Smirnov and Cramér-von Mises type statistics, respectively, given by

$$
\sup _{\mathbf{u} \in[0,1]^{d}}\left|\mathbb{A}_{n}(\mathbf{u})\right| \text {, and } \int_{[0,1]^{d}} \mathbb{A}_{n}^{2}(\mathbf{u}) d \mathbf{u} \text {. }
$$

We get

$$
\left|\sup _{\mathbf{u} \in[0,1]^{d}}\right| \mathbb{A}_{n}(\mathbf{u})\left|-\sup _{\mathbf{u} \in[0,1]^{d}} \frac{1}{n^{1 / 2}}\right| \mathscr{K}_{\mathbb{C}}^{*}(\mathbf{u}, n) \mid=O\left(n^{-1 /(4 d)}(\log n)^{3 / 2}\right),
$$

and

$$
\left|\int_{[0,1]^{d}} \mathbb{A}_{n}^{2}(\mathbf{u}) d \mathbf{u}-\frac{1}{n} \int_{[0,1]^{d}} \mathscr{K}_{\mathbb{C}}^{* 2}(\mathbf{u}, n) d \mathbf{u}\right|=O\left(n^{-1 /(4 d)}(\log n)^{3 / 2}(\log \log n)^{1 / 2}\right) .
$$

At this point, we mention that the proof of (2.6) closely follows the lines of Bouzebda et al. (2011b) and Bouzebda et al. (2011a) among others. Therefore, we omit the details. Another interesting application of the approximation of $\mathbb{A}_{n}(\mathbf{u})$ in terms of Gaussian process in both $\mathbf{u}$ and $n$ is the change-point problem, as in Rémillard (2010) and Csörgó et al. (1997), and leaves this study open for future research.

Note that the covariance structure of the process $\left\{\mathscr{K}_{\mathbb{C}}^{*}(\mathbf{u}, n): \mathbf{u} \in[0,1]^{d}, n \geq 1\right\}$ depends on the first derivatives of the copula $\mathbb{C}(\cdot)$ which are, in general, unknown in practice. To circumvent this problem, one can use a multiplier central limit theorem, please refer to van der Vaart and Wellner (1996), as suggested in Rémillard and Scaillet (2009), Scaillet (2005) and recently Rémillard (2010). We mention that the usual bootstrap based on resampling was proposed in Fermanian et al. (2004). Here, for easy reference and completeness, we recall the procedure given in Rémillard and Scaillet (2009), which is more appropriate for our setting. Let $N$ be a large integer, and let $Z_{i}^{(k)}, i=1, \ldots, n, k=1, \ldots, N$, be i.i.d. random variables with mean 0 and variance 1 , independent of the data $\mathbf{U}_{1}, \ldots, \mathbf{U}_{n}$. Moreover, for any $k=1, \ldots, N$, let

$$
\begin{aligned}
\boldsymbol{\alpha}_{n}^{(k)}(\mathbf{u}) & :=\frac{1}{\sqrt{n}} \sum_{i=1}^{n} Z_{i}^{(k)}\left\{\mathbb{1}\left\{G_{1 n}\left(U_{1 i}\right) \leq u_{1}, \ldots, G_{d n}\left(U_{d i}\right) \leq u_{d}\right\}-\overline{\mathbb{C}}_{n}(\mathbf{u})\right\} \\
& =\frac{1}{\sqrt{n}} \sum_{k=1}^{n}\left(Z_{i}^{(k)}-\bar{\varepsilon}_{n}\right) \mathbb{1}\left\{G_{1 n}\left(U_{1 i}\right) \leq u_{1}, \ldots, G_{d n}\left(U_{d i}\right) \leq u_{d}\right\},
\end{aligned}
$$

where

$$
\overline{\mathbb{C}}_{n}(\mathbf{u}):=\frac{1}{n} \sum_{i=1}^{n} \mathbb{1}\left\{G_{1 n}\left(U_{1 i}\right) \leq u_{1}, \ldots, G_{d n}\left(U_{d i}\right) \leq u_{d}\right\},
$$

and $\bar{\varepsilon}_{n}=\frac{1}{n} \sum_{i=1}^{n} \varepsilon_{i}$, and for any $j=1, \ldots, d$,

$$
\begin{aligned}
\alpha_{j n}^{(k)}\left(u_{j}\right) & :=\boldsymbol{\alpha}_{n}^{(k)}\left(1, \ldots, 1, u_{j}, 1, \ldots, 1\right) \\
& =\frac{1}{\sqrt{n}} \sum_{i=1}^{n}\left(Z_{i}^{(k)}-\bar{\varepsilon}_{n}\right) \mathbb{1}\left\{G_{j n}\left(U_{j i}\right) \leq u_{j}\right\} .
\end{aligned}
$$

Finally, for all $\mathbf{u} \in[0,1]^{d}$, and for all $k=1, \ldots, N$, let

$$
\mathbb{A}_{n}^{(k)}(\mathbf{u}):=\boldsymbol{\alpha}_{n}^{(k)}(\mathbf{u})-\sum_{j=1}^{d} \alpha_{j n}^{(k)}\left(u_{j}\right) \mathbb{C}_{n}^{[j]}(\mathbf{u}),
$$


where $\mathbb{C}_{n}^{[j]}(\mathbf{u})$ is a consistent estimator of the partial derivative $\frac{\partial \mathbb{C}(\mathbf{u})}{\partial u_{j}}$, for $j=1, \ldots, d$, and given, as in Rémillard and Scaillet (2009), by

$$
\mathbb{C}_{n}^{[j]}(\mathbf{u}):=\frac{\mathbb{C}_{n}\left(\mathbf{u}+h e_{j}\right)-\mathbb{C}_{n}\left(\mathbf{u}-h e_{j}\right)}{2 h},
$$

where $e_{j}$ is the $j$-th column of the $d \times d$ identity matrix. From Theorem 2.1 in Rémillard and Scaillet (2009), a practical choice of $h$ is $n^{-1 / 2}$. We will not investigate the question of the strong approximations of the processes $\mathbb{A}_{n}^{(k)}(\cdot)$ in the present paper.

Remark 2.5 In Bücher and Dette (2010), a new procedure, which has the most attractive theoretical properties, has been proposed to circumvent the problem of the estimation of the derivatives of the unknown copula. In the same paper, the finite-sample properties of some methods are compared in a simulation study, and the multiplier approach, by Rémillard and Scaillet (2009), yields the best results in most cases.

Recently, Rémillard and Scaillet (2009) considered the two sample problem in the copula setting. Let $\mathbb{C}_{n}(\cdot)$ and $\mathbb{D}_{m}(\cdot)$ denote the empirical copulas functions based on independent samples of sizes $n$ and $m$, respectively. Theorem 2.1 may be used for statistical comparison procedures of the unknown copulas $\mathbb{C}(\cdot)$ and $\mathbb{D}(\cdot)$ based on $\mathbb{C}_{n}(\cdot)$ and $\mathbb{D}_{m}(\cdot)$. Consider the empirical process $\mathbb{A}_{n ; m}(\cdot)$, defined by

$$
\mathbb{A}_{n ; m}(\mathbf{u}):=\left\{\frac{n m}{n+m}\right\}^{1 / 2}\left\{\mathbb{C}_{n}(\mathbf{u})-\mathbb{D}_{m}(\mathbf{u})\right\}, \text { for } \mathbf{u} \in[0,1]^{d}
$$

In order to test the null hypothesis $\mathscr{H}_{0}: \mathbb{C}=\mathbb{D}$, we use Cramér-von Mises type statistic, given by

$$
\Omega_{n ; m}:=\int_{[0,1]^{d}}\left\{\mathbb{A}_{n ; m}(\mathbf{u})\right\}^{2} d \mathbf{u} .
$$

We need to define the following Gaussian process

$$
\mathscr{K}_{n ; m}^{*}(\mathbf{u}):=[m /(n+m)]^{1 / 2} \frac{1}{n^{1 / 2}} \mathscr{K}_{\mathbb{C}}^{*}(\mathbf{u}, n)+[n /(n+m)]^{1 / 2} \frac{1}{m^{1 / 2}} \mathscr{K}_{\mathbb{D}}^{*}(\mathbf{u}, m) .
$$

Using Theorem 2.1, one can show, as $\min (n, m) \rightarrow \infty$ and $n /(n+m) \rightarrow \lambda \in[0,1]$, then we have almost surely,

$$
\begin{aligned}
& \left|\int_{[0,1]^{d}}\left\{\mathbb{A}_{n ; m}(\mathbf{u})\right\}^{2} d \mathbf{u}-\int_{[0,1]^{d}}\left\{\mathscr{K}_{n ; m}^{*}(\mathbf{u})\right\}^{2} d \mathbf{u}\right| \\
& \quad=O\left(\max \left(\frac{(\log n)^{3 / 2}}{n^{1 /(4 d)}}, \frac{(\log m)^{3 / 2}}{m^{1 /(4 d)}}\right) \times \max \left((\log \log n)^{1 / 2},(\log \log m)^{1 / 2}\right)\right) .
\end{aligned}
$$

\section{Applications}

\subsection{Smoothed empirical copula processes}

A seemingly natural kernel-type estimator $\widehat{\mathbb{C}}_{n}(\cdot)$ of $\mathbb{C}(\cdot)$ would be

$$
\widehat{\mathbb{C}}_{n}(\mathbf{u}):=\frac{1}{h} \int_{[0,1]^{d}} k\left(\frac{\mathbf{u}-\mathbf{v}}{h^{1 / d}}\right) \mathbb{C}_{n}(\mathbf{v}) d \mathbf{v}, \text { for } \mathbf{u} \in[0,1]^{d},
$$


where $k(\cdot)$ is a kernel function and $h=h(n)$ is the smoothing parameter. For notational convenience, we have chosen the same bandwidth sequence for each margins. This assumption can be dropped easily. The kernel estimation of copula function is a rich topic of researches, we only mention Omelka et al. (2009), Chen and Huang (2007) and Fermanian et al. (2004), see their lists of references for related studies. As in the previous section, we define the smoothed empirical copulas process, for $n \geq 1$, by

$$
\widehat{\mathbb{A}}_{n}(\mathbf{u}):=\sqrt{n}\left(\widehat{\mathbb{C}}_{n}(\mathbf{u})-\mathbb{C}(\mathbf{u})\right), \text { for } \mathbf{u} \in[0,1]^{d}
$$

We will describe the asymptotic properties of the smoothed empirical copulas process $\left\{\widehat{\mathbb{A}}_{n}(\mathbf{u}): \mathbf{u} \in\right.$ $\left.[0,1]^{d} ; n>0\right\}$ under the following assumptions.

(F.1) There exists a constant $0<\mathfrak{C}<\infty$ such that

$$
\sup _{\mathbf{u} \in[0,1]^{d}}\left|\frac{\partial^{s} \mathbb{C}(\mathbf{u})}{\partial^{j_{1}} u_{1} \ldots \partial^{j_{d}} u_{d}}\right| \leq \mathfrak{C}, \quad j_{1}+\cdots+j_{d}=s .
$$

Suppose that $\{h(n)\}_{n \geq 1}$ is a sequence of positive constants which satisfies the following condition.

(C.1) $h=h(n) \rightarrow 0, n h \rightarrow \infty$ and $\sqrt{n} h^{s / d} \rightarrow 0$ as $n \rightarrow \infty$.

The following conditions on the kernel function $k(\cdot)$ are assumed in our analysis.

(C.2) $k(\cdot)$ is a continuous density function and compactly supported;

(C.3) $k(\cdot)$ is of order $s$, i.e.,

$$
\begin{aligned}
& \int_{\mathbb{R}^{d}} k(\mathbf{u}) d \mathbf{u}=1 \\
& \int_{\mathbb{R}^{d}} u_{1}^{j_{1}} \ldots u_{d}^{j_{d}} k(\mathbf{u}) d \mathbf{u}=0, \quad j_{1}, \ldots, j_{d} \geq 0, \quad j_{1}+\cdots+j_{d}=1, \ldots, s-1, \\
& \int_{\mathbb{R}^{d}}\left|u_{1}^{j_{1}} \ldots u_{d}^{j_{d}}\right| k(\mathbf{u}) d \mathbf{u}<\infty, \quad j_{1}, \ldots, j_{d} \geq 0, \quad j_{1}+\cdots+j_{d}=s .
\end{aligned}
$$

It is now possible to state the main theoretical result of this section which provides the limiting behavior of the smoothed empirical copulas process $\left\{\widehat{\mathbb{A}}_{n}(\mathbf{u}): \mathbf{u} \in[0,1]^{d} ; n>0\right\}$.

Corollary 3.1 Assume that (F.1) and (C.1)-(C.3) hold. Then, on a suitable probability space, we may define the smoothed empirical copula processes $\left\{\widehat{\mathbb{A}}_{n}(\mathbf{u}): \mathbf{u} \in[0,1]^{d} ; n>0\right\}$ in combination with the Gaussian process $\left\{\mathscr{K}_{\mathbb{C}}^{*}(\mathbf{u}, t): \mathbf{u} \in[0,1]^{d} ; t \geq 0\right\}$, in such a way that, as $n \rightarrow \infty$

$$
\sup _{\mathbf{u} \in[0,1]^{d}}\left|\widehat{\mathbb{A}}_{n}(\mathbf{u})-\frac{1}{\sqrt{n}} \mathscr{K}_{\mathbb{C}}^{*}(\mathbf{u}, n)\right|=o_{\mathbb{P}}(1) .
$$

The proof of Corollary 3.1 is postponed until Section 4.

The result of Corollary 3.1 is motivated by the following remark. 
Remark 3.2 The empirical copula provides a universal way for estimation purposes. Unfortunately, its discontinuous feature induces some difficulties: the graphical representations of the copula may not be satisfactory from a visual and intuitive point of view. Moreover, there is no unique choice for building the inverse function of marginal functions. Finally, since the empirical copula estimator is not differentiable, it cannot, for example, be used for optimization purposes. Studies have shown that a smoothed estimator may be preferable to the sample estimator. First, smoothing reduces the random variation in the data, resulting in a more efficient estimator. Second, smoothing gives a smooth curve that displays some interesting features. For more details on the subject we may refer to Chen and Huang (2007).

Remark 3.3 1. Corollary 3.1 remains valid when replacing the condition that the kernel function $k(\cdot)$ having compact support in (C.2) by another condition (C.4) which content is as follows

(C.4) There exists a sequence of positive real numbers $a_{n}$ such that $a_{n} h$ tends to zero when $n$ tends to infinity, and

$$
\sqrt{n} \int_{\left\{\|\mathbf{v}\|>a_{n}\right\}}|k(\mathbf{v})| d \mathbf{v} \rightarrow 0 .
$$

2. Note that the conditions of Corollary 3.1 are grouped to control the deviations between the normalized empirical copula process $\left\{\mathbb{A}_{n}(\mathbf{u}): \mathbf{u} \in[0,1]^{d} ; n>0\right\}$ and the smoothed empirical copula process $\left\{\widehat{\mathbb{A}}_{n}(\mathbf{u}): \mathbf{u} \in[0,1]^{d} ; n>0\right\}$.

\subsection{The law of iterated logarithm for the normalized empirical copula process}

From Theorem 2.1, we have almost surely

$$
\limsup _{n \rightarrow \infty}\left\{\left(\frac{n}{2 \log \log n}\right)^{1 / 2} \sup _{\mathbf{u} \in[0,1]^{d}}\left|\mathbb{C}_{n}(\mathbf{u})-\mathbb{C}(\mathbf{u})\right|\right\}=\limsup _{n \rightarrow \infty} \frac{\sup _{\mathbf{u} \in[0,1]^{d}}\left|\mathscr{K}_{\mathbb{C}}^{*}(\mathbf{u}, n)\right|}{(2 n \log \log n)^{1 / 2}}
$$

Note that (3.4) readily implies the following corollary, which is a straightforward consequence of Theorem 2.1 .

Corollary 3.4 Under the same conditions of Theorem 2.1, we have

$$
\limsup _{n \rightarrow \infty}\left\{\left(\frac{n}{2 \log \log n}\right)^{1 / 2} \sup _{\mathbf{u} \in[0,1]^{d}}\left|\mathbb{C}_{n}(\mathbf{u})-\mathbb{C}(\mathbf{u})\right|\right\}=\rho, \text { a.s. }
$$

where

$$
\rho^{2}:=\sup _{\mathbf{u} \in[0,1]^{d}} \operatorname{Var}\left(\mathscr{K}_{\mathbb{C}}^{*}(\mathbf{u}, 1)\right)
$$

Remark 3.5 A result similar to Corollary 3.4 was obtained by Deheuvels (1979) (refer to Theorem 3.1) using a different method. 
Remark 3.6 Statistics of the form

$$
\mathbf{R}_{n}:=\frac{1}{n} \sum_{i=1}^{n} J\left(G_{1 n}\left(X_{1 i}\right), \ldots, G_{d n}\left(X_{d i}\right)\right),
$$

belong to the general class of multivariate rank statistics. Their asymptotic properties have been investigated at length by a number of authors, among whom we may quote Ruymgaart et al. (1972), Rüschendorf $(1974,1976)$. In particular, the previous authors have provided regularity conditions, imposed on $J(\cdot)$, which imply the asymptotic normality of $\mathbf{R}_{n}$. It is easy to see that

$$
\mathbf{R}_{n}=\int_{[0,1]^{d}} J(\mathbf{u}) d \overline{\mathbb{C}}_{n}(\mathbf{u}) .
$$

Since the difference between $\overline{\mathbb{C}}_{n}(\cdot)$ and $\mathbb{C}_{n}(\cdot)$ is negligible, see Fermanian et al. (2004) or Deheuvels (2009), the asymptotic normality of $\mathbf{R}_{n}$ can be established under the weakest set of assumptions (see, Theorem 6 in Fermanian et al. (2004)) using Theorem 2.1.

\section{Proofs}

This section is devoted to the proofs of our results.

\section{Proof of Theorem 2.1.}

Consider the empirical processes defined, respectively, for $n \geq 1, \mathbf{u} \in[0,1]^{d}$ and $0 \leq u_{j} \leq 1$, for $j=1, \ldots, d$, by

$$
\begin{aligned}
\boldsymbol{\alpha}_{n}(\mathbf{u}) & :=n^{1 / 2}\left(\mathbb{G}_{n}(\mathbf{u})-\mathbb{C}(\mathbf{u})\right), \\
\alpha_{j n}\left(u_{j}\right) & :=n^{1 / 2}\left\{G_{j n}\left(u_{j}\right)-u_{j}\right\}, \\
\beta_{j n}\left(u_{j}\right) & :=n^{1 / 2}\left\{G_{j n}^{-}\left(u_{j}\right)-u_{j}\right\} .
\end{aligned}
$$

Keep in mind the definition (1.10) of $\mathbb{A}_{n}(\cdot)$. The normalized empirical copula process can be written, for $\mathbf{u} \in[0,1]^{d}$, as follows

$$
\begin{aligned}
& \mathbb{A}_{n}(\mathbf{u})=n^{1 / 2}\left(\mathbb{G}_{n}\left(G_{1, n}^{-}\left(u_{1}\right), \ldots, G_{d, n}^{-}\left(u_{d}\right)\right)-\mathbb{C}\left(u_{1}, \ldots, u_{d}\right)\right) \\
&=\boldsymbol{\alpha}_{n}\left(G_{1, n}^{-}\left(u_{1}\right), \ldots, G_{d, n}^{-}\left(u_{d}\right)\right)+n^{1 / 2}\left\{\mathbb{C}\left(G_{1, n}^{-}\left(u_{1}\right), \ldots, G_{d, n}^{-}\left(u_{d}\right)\right)\right. \\
&\left.\quad \quad-\mathbb{C}\left(u_{1}, \ldots, u_{d}\right)\right\} \\
&=\quad \boldsymbol{\alpha}_{n}\left(u_{1}+n^{-1 / 2} \beta_{1 n}\left(u_{1}\right), \ldots, u_{d}+n^{-1 / 2} \beta_{d n}\left(u_{d}\right)\right) \\
&\left.\quad+n^{1 / 2}\left\{\mathbb{C}\left(u_{1}+n^{-1 / 2} \beta_{1 n}\left(u_{1}\right), \ldots, u_{d}+n^{-1 / 2} \beta_{d n}\left(u_{d}\right)\right)-\mathbb{C}\left(u_{1}, \ldots, u_{d}\right)\right)\right\} \\
&=\quad \boldsymbol{\alpha}_{n}(\mathbf{u})+\left\{\boldsymbol{\alpha}_{n}\left(\mathbf{u}+n^{-1 / 2} \beta_{n}(\mathbf{u})\right)-\boldsymbol{\alpha}_{n}(\mathbf{u})\right\} \\
& \quad+n^{1 / 2}\left\{\mathbb{C}\left(\mathbf{u}+n^{-1 / 2} \beta_{n}(\mathbf{u})\right)-\mathbb{C}(\mathbf{u})\right\} \\
&= \boldsymbol{\alpha}_{n}(\mathbf{u})+\boldsymbol{\Delta}_{1}(\mathbf{u}, n)+\boldsymbol{\Delta}_{2}(\mathbf{u}, n),
\end{aligned}
$$


where $\left(\mathbf{u}+n^{-1 / 2} \beta_{n}(\mathbf{u})\right)=\left(u_{1}+n^{-1 / 2} \beta_{1 n}\left(u_{1}\right), \ldots, u_{d}+n^{-1 / 2} \beta_{d n}\left(u_{d}\right)\right)$. The decomposition (4.4) is the main key to our proof. We first compute the right side term $\Delta_{2}(\cdot, n)$ of (4.4). Under differentiability assumption on $\mathbb{C}(\cdot)$ and by successive Taylor expansions, we readily obtain the equality

$$
\begin{aligned}
\boldsymbol{\Delta}_{2}(\mathbf{u}, n)= & \sum_{j=1}^{d} \frac{\partial \mathbb{C}(\mathbf{u})}{\partial u_{j}} \sqrt{n}\left(G_{n j}^{-}\left(u_{j}\right)-u_{j}\right) \\
& +\frac{\sqrt{n}}{2} \sum_{j=1}^{d} \sum_{k=1}^{d} \frac{\partial^{2} \mathbb{C}\left(\mathbf{u}^{*}\right)}{\partial u_{j} \partial u_{k}}\left(G_{n j}^{-}\left(u_{j}\right)-u_{j}\right)\left(G_{n k}^{-}\left(u_{k}\right)-u_{k}\right),
\end{aligned}
$$

which holds for some point $\mathbf{u}^{*}$ in the interior of the line segment joining $\left(G_{n 1}^{-}\left(u_{1}\right), \ldots, G_{n d}^{-}\left(u_{d}\right)\right)$ and $\left(u_{1}, \ldots, u_{d}\right)$. It follows from the definition of $\boldsymbol{\alpha}_{n}(\cdot)$ in $(4.1)$, for $u_{j} \in[0,1], j=1, \ldots, d$, that

$$
\begin{aligned}
\sqrt{n}\left(G_{n j}^{-}\left(u_{j}\right)-u_{j}\right) & =-\sqrt{n}\left(G_{n j}\left(G_{n j}^{-}\left(u_{j}\right)\right)-G_{n j}^{-}\left(u_{j}\right)\right)+\sqrt{n}\left(G_{n j}\left(G_{n j}^{-}\left(u_{j}\right)\right)-u_{j}\right) \\
& =-\boldsymbol{\alpha}_{n}\left(\mathbf{1}, G_{n j}^{-}\left(u_{j}\right), \mathbf{1}\right)+\sqrt{n}\left(G_{n j}\left(G_{n j}^{-}\left(u_{j}\right)\right)-u_{j}\right) .
\end{aligned}
$$

Using the fact, for $u_{j} \in[0,1], j=1, \ldots, d$, that

$$
\left|G_{n j}\left(G_{n j}^{-}\left(u_{j}\right)\right)-u_{j}\right| \leq \frac{1}{n}
$$

and the Chung (1949)'s law of the iterated logarithm, one finds, almost surely,

$$
\boldsymbol{\Delta}_{2}(\mathbf{u}, n)=-\sum_{j=1}^{d} \frac{\partial \mathbb{C}(\mathbf{u})}{\partial u_{j}} \boldsymbol{\alpha}_{n}\left(\mathbf{1}, G_{n j}^{-}\left(u_{j}\right), \mathbf{1}\right)+O\left(n^{-1 / 2} \log \log n\right),
$$

uniformly in $\mathbf{u} \in[0,1]^{d}$. It is well known from Stute's work [Stute (1982), p. 99], that we have, almost surely, for $n$ sufficiently large and $j=1, \ldots, d$,

$$
\sup _{u_{j} \in[0,1]}\left|\boldsymbol{\alpha}_{n}\left(\mathbf{1}, G_{n j}^{-}\left(u_{j}\right), \mathbf{1}\right)-\boldsymbol{\alpha}_{n}\left(\mathbf{1}, u_{j}, \mathbf{1}\right)\right|=O\left(n^{-1 / 4}(\log n)^{1 / 2}(\log \log n)^{1 / 4}\right) .
$$

Then, it follows that uniformly in $\mathbf{u} \in[0,1]^{d}$, almost surely, for $n$ sufficiently large

$$
\boldsymbol{\Delta}_{2}(\mathbf{u}, n)=-\sum_{j=1}^{d} \frac{\partial \mathbb{C}(\mathbf{u})}{\partial u_{j}} \boldsymbol{\alpha}_{n}\left(\mathbf{1}, u_{j}, \mathbf{1}\right)+O\left(n^{-1 / 4}(\log n)^{1 / 2}(\log \log n)^{1 / 4}\right),
$$

as was observed by Stute (1984), p. 371. We next evaluate the term $\Delta_{1}(\cdot, n)$ in the right hand side of (4.4). Recall that $\boldsymbol{\Delta}_{1}(\mathbf{u}, n)$ is the difference between $\boldsymbol{\alpha}_{n}\left(\mathbf{u}+n^{-1 / 2} \beta_{n}(\mathbf{u})\right)$ and $\boldsymbol{\alpha}_{n}(\mathbf{u})$. Let $w_{n}(\cdot)$ be the modulus of continuity of $\boldsymbol{\alpha}_{n}(\cdot)$, that is

$$
w_{n}(\mathbf{a}):=\sup \left\{\alpha_{n}(A): A=\prod_{j=1}^{d}\left[u_{j}, v_{j}\right] \in[0,1]^{d}, \text { with }\left|\left[u_{j}, v_{j}\right]\right|=v_{j}-u_{j} \leq a_{j}, \forall j=1, \ldots, d\right\},
$$

where $\mathbf{a}:=\left(a_{1}, \ldots, a_{d}\right)$. We will make use of the following fact which is a particular case of Theorem 2.1, p. 367 of Stute (1984).

Fact 1. Let $\left\{a_{n}\right\}_{n \geq 1}$ be a sequence in $(0,1)$ such as $a_{n} \downarrow 0$, as $n \rightarrow \infty$, and

$$
\text { i) } \left.n a_{n}^{d} \uparrow \infty, \quad \text { ii) } n a_{n}^{d} / \log n \rightarrow \infty, \quad \text { iii }\right) \log \left(1 / a_{n}\right) / \log \log n \rightarrow \infty .
$$


Then, we have, almost surely,

$$
\lim _{n \rightarrow \infty}\left\{2 a_{n}^{d} \log \left(1 / a_{n}^{d}\right)\right\}^{-1 / 2} w_{n}\left(a_{n}, \ldots, a_{n}\right)=1 .
$$

Once more, an application of the Chung (1949) law of the iterated logarithm shows that, for each $j=1, \ldots, d$, almost surely,

$$
\lim \sup _{n \rightarrow \infty}\left\{(\log \log n)^{-1 / 2} \sup _{0 \leq u_{j} \leq 1}\left|\beta_{j n}\left(u_{j}\right)\right|\right\}=2^{-1 / 2} .
$$

In view of (4.6), we have almost surely, for all $j=1, \ldots, d$ and $n$ large enough,

$$
\begin{aligned}
\sup _{0 \leq u_{j} \leq 1}\left|n^{-1 / 2} \beta_{j n}\left(u_{j}\right)\right| & \leq \frac{(\log \log n)^{1 / 2}}{n^{1 / 2}} \\
& \leq \frac{(\log n)^{2 / d}}{n^{1 / d}}:=a_{n},
\end{aligned}
$$

an application of Fact 1 shows that, as $n \rightarrow \infty$, we have, almost surely,

$$
\sup _{\mathbf{u} \in[0,1]^{d}}\left|\boldsymbol{\Delta}_{1}(\mathbf{u}, n)\right| \leq w_{n}\left(\mathbf{a}_{n}\right)=O\left(n^{-1 / 2}(\log n)^{3 / 2}\right)
$$

where $\mathbf{a}_{n}:=\left(a_{n}, \ldots, a_{n}\right)$. The next fact, due to Csörgó and Horváth (1988), p. 102, provides a strong approximation result appropriate to our need. Recall the definitions (2.2) and (4.1).

Fact 2. On a suitable probability space $(\Omega, \mathscr{A}, \mathbb{P})$, it is possible to define $\left\{\boldsymbol{\alpha}_{n}(\mathbf{u}): \mathbf{u} \in[0,1]^{d}\right\}$, jointly with the sequence of Gaussian processes $\left\{\mathscr{K}_{\mathbb{C}}(\mathbf{u}, t): \mathbf{u} \in[0,1]^{d}, t \geq 0\right\}$, in such a way that, as $n \rightarrow \infty$, almost surely,

$$
\sup _{\mathbf{u} \in[0,1]^{d}}\left|\sqrt{n} \boldsymbol{\alpha}_{n}(\mathbf{u})-\mathscr{K}_{\mathbb{C}}(\mathbf{u}, n)\right|=O\left(n^{1 / 2-1 /(4 d)}(\log n)^{3 / 2}\right) .
$$

In view of the above Fact, by combining (4.5) and (4.7) with the triangle inequality, we readily obtain

$$
\begin{aligned}
\sup _{\mathbf{u} \in[0,1]^{d}} & \left|\sqrt{n} \mathbb{A}_{n}(\mathbf{u})-\mathscr{K}_{\mathbb{C}}^{*}(\mathbf{u}, n)\right| \leq \sup _{\mathbf{u} \in[0,1]^{d}}\left|\sqrt{n} \boldsymbol{\alpha}_{n}(\mathbf{u})-\mathscr{K}_{\mathbb{C}}(\mathbf{u}, n)\right|+\sqrt{n} w_{n}\left(\mathbf{a}_{n}\right) \\
& +\sum_{j=1}^{d}\left|\frac{\partial \mathbb{C}(\mathbf{u})}{\partial u_{j}}\right| \sup _{0 \leq u_{j} \leq 1}\left|\sqrt{n} \boldsymbol{\alpha}_{n}\left(\mathbf{1}, u_{j}, \mathbf{1}\right)-\mathscr{K}_{\mathbb{C}}^{(j)}\left(\mathbf{1}, u_{j}, \mathbf{1}, n\right)\right| \\
& +O\left(n^{1 / 4}(\log n)^{1 / 2}(\log \log n)^{1 / 4}\right), \\
\leq \quad & O\left(n^{1 / 2-1 /(4 d)}(\log n)^{3 / 2}\right)+O\left((\log n)^{3 / 2}\right) \\
& +O\left(n^{1 / 2-1 /(4 d)}(\log n)^{3 / 2}\right)+O\left(n^{1 / 4}(\log n)^{1 / 2}(\log \log n)^{1 / 4}\right) \\
= & O\left(n^{1 / 2-1 /(4 d)}(\log n)^{3 / 2}\right) .
\end{aligned}
$$

Note that we have used the fact that the first-order partial derivatives of a copula are bounded (see Theorem 2.2.7 of Nelsen (2006)). Then, we have almost surely, for all $n$ sufficiently large,

$$
\sup _{\mathbf{u} \in[0,1]^{d}}\left|\sqrt{n} \mathbb{A}_{n}(\mathbf{u})-\mathscr{K}_{\mathbb{C}}^{*}(\mathbf{u}, n)\right|=O\left(n^{1 / 2-1 /(4 d)}(\log n)^{3 / 2}\right)
$$

and thus the proof of Theorem 2.1 is completed. 


\section{Proof of Corollary 3.1.}

We shall first study the behavior of the difference between the normalized empirical copula process $\mathbb{A}_{n}(\cdot)$ and the smoothed empirical copula process $\widehat{\mathbb{A}}_{n}(\cdot)$. Recall the definition (3.1). Notice that, for each $\mathbf{u} \in[0,1]^{d}$,

$$
\begin{aligned}
\widehat{\mathbb{A}}_{n}(\mathbf{u})= & \sqrt{n}\left(\widehat{\mathbb{C}}_{n}(\mathbf{u})-\mathbb{C}(\mathbf{u})\right) \\
= & \sqrt{n}\left(\frac{1}{h} \int_{[0,1]^{d}} k\left(\frac{\mathbf{u}-\mathbf{v}}{h^{1 / d}}\right) \mathbb{C}_{n}(\mathbf{v}) d \mathbf{v}-\mathbb{C}(\mathbf{u})\right) \\
= & \left(\frac{1}{h} \int_{[0,1]^{d}} k\left(\frac{\mathbf{u}-\mathbf{v}}{h^{1 / d}}\right) \sqrt{n}\left(\mathbb{C}_{n}(\mathbf{v})-\mathbb{C}(\mathbf{v})\right)\right) d \mathbf{v} \\
& +\sqrt{n}\left(\frac{1}{h} \int_{[0,1]^{d}} k\left(\frac{\mathbf{u}-\mathbf{v}}{h^{1 / d}}\right) \mathbb{C}(\mathbf{v}) d \mathbf{v}-\mathbb{C}(\mathbf{u})\right) \\
= & \left(\frac{1}{h} \int_{[0,1]^{d}} k\left(\frac{\mathbf{u}-\mathbf{v}}{h^{1 / d}}\right) \mathbb{A}_{n}(\mathbf{v})\right) d \mathbf{v} \\
& +\sqrt{n}\left(\frac{1}{h} \int_{[0,1]^{d}} k\left(\frac{\mathbf{u}-\mathbf{v}}{h^{1 / d}}\right) \mathbb{C}(\mathbf{v}) d \mathbf{v}-\mathbb{C}(\mathbf{u})\right) .
\end{aligned}
$$

We will make use of the following straightforward inequality

$$
\begin{aligned}
\sup _{\mathbf{u} \in[0,1]^{d}} & \left|\widehat{\mathbb{A}}_{n}(\mathbf{u})-\mathbb{A}_{n}(\mathbf{u})\right| \\
\leq & \sup _{\mathbf{u} \in[0,1]^{d}}\left|\int_{\prod_{i=1}^{d}\left[\frac{u_{i}-1}{h^{1 / d}}, \frac{u_{i}}{h^{1 / d}}\right]}\left(\mathbb{A}_{n}\left(\mathbf{u}-h^{1 / d} \mathbf{v}\right)-\mathbb{A}_{n}(\mathbf{u})\right) k(\mathbf{v}) d \mathbf{v}\right| \\
& +\sup _{\mathbf{u} \in[0,1]^{d}}\left|\mathbb{A}_{n}(\mathbf{u})\right|\left|\int_{\prod_{i=1}^{d}\left[\frac{u_{i}-1}{h^{1 / d}}, \frac{u_{i}}{h^{1 / d}}\right]} k(\mathbf{v}) d \mathbf{v}-1\right| \\
& +\sqrt{n} \sup _{\mathbf{u} \in[0,1]^{d}}\left|\int_{\prod_{i=1}^{d}\left[\frac{u_{i}-1}{h^{1 / d}}, \frac{u_{i}}{h^{1 / d}}\right]}\left(\mathbb{C}\left(\mathbf{u}-h^{1 / d} \mathbf{v}\right)-\mathbb{C}(\mathbf{u})\right) k(\mathbf{v}) d \mathbf{v}\right| \\
& +\sqrt{n} \sup _{\mathbf{u} \in[0,1]^{d}}|\mathbb{C}(\mathbf{u})|\left|\int_{\prod_{i=1}^{d}\left[\frac{u_{i}-1}{h^{1 / d}}, \frac{u_{i}}{h^{1 / d}}\right]} k(\mathbf{v}) d \mathbf{v}-1\right| \\
:= & \nabla_{1 ; n}+\nabla_{2 ; n}+\nabla_{3 ; n}+\nabla_{4 ; n} .
\end{aligned}
$$

We first evaluate $\nabla_{3 ; n}$ in the right side of (4.10). Under conditions (F.1), (C.1)-(C.3) and applying a Taylor series expansion of order $s$, we can see that

$$
\nabla_{3 ; n}=\frac{h^{s / d}}{s !} \sqrt{n} \sup _{\mathbf{u} \in[0,1]^{d}}\left|\int \sum_{j_{1}+\cdots+j_{d}=s} u_{1}^{j_{1}} \ldots u_{d}^{j_{d}} \frac{\partial^{s} \mathbb{C}\left(\mathbf{u}-h_{n} \theta \mathbf{v}\right)}{\partial u_{1}^{j_{1}} \ldots \partial u_{d}^{j_{d}}} k(\mathbf{v}) d \mathbf{v}\right|,
$$

where $\theta=\left(\theta_{1}, \ldots, \theta_{d}\right)$ and $0<\theta_{j}<1$, for $j=1, \ldots, d$. Thus, a straightforward application of Lebesgue dominated convergence theorem gives

$$
n^{-1 / 2} h^{-(s / d)} \nabla_{3 ; n}=\frac{1}{k !} \sup _{\mathbf{u} \in[0,1]^{d}}\left|\sum_{j_{1}+\cdots+j_{d}=s} \frac{\partial^{s} \mathbb{C}(\mathbf{u})}{\partial u_{1}^{j_{1}} \ldots \partial u_{d}^{j_{d}}} \int u_{1}^{j_{1}} \ldots u_{d}^{j_{d}} k(\mathbf{v}) d \mathbf{v}\right| .
$$


Then by condition (C.1) and (C.3), we conclude that, for all $n$ sufficiently large,

$$
\nabla_{3 ; n}=O\left(n^{1 / 2} h^{s / d}\right)=o(1) .
$$

Making use of Theorem 2.1 in connection with the almost sure continuity of the Gaussian process $\left\{\mathscr{K}_{\mathbb{C}}^{*}(\mathbf{u}, t): \mathbf{u} \in[0,1]^{d} ; t \geq 0\right\}$, we have, for all $n$ sufficiently large,

$$
\begin{aligned}
\nabla_{1 ; n} & \leq \sup _{\mathbf{u}, \mathbf{v} \in[0,1]^{d}|\mathbf{u}-\mathbf{v}| \leq h} \sup _{n}\left|\mathbb{A}_{n}(\mathbf{v})-\mathbb{A}_{n}(\mathbf{u})\right|\left|\int k(\mathbf{v}) d \mathbf{v}\right| \\
& =o_{\mathbb{P}}(1) O(1)=o_{\mathbb{P}}(1) .
\end{aligned}
$$

We will next evaluate $\nabla_{2 ; n}$ in the right side of (4.10). We have

$$
\sup _{\mathbf{u} \in[0,1]^{d}}\left|\mathbb{A}_{n}(\mathbf{u})\right|=O_{\mathbb{P}}(1)
$$

and as $n$ tends to infinity, by condition (C.2), we conclude that

$$
\sqrt{n}\left|\int_{\prod_{i=1}^{d}\left[\frac{u_{i}-1}{h^{1 / d}}, \frac{u_{i}}{h^{1 / d}}\right]} k(\mathbf{v}) d \mathbf{v}-1\right|=o(1) .
$$

Then we obtain

$$
\nabla_{2 ; n}=o_{\mathbb{P}}(1) .
$$

As we closely follow the lines of the proof of $\nabla_{2 ; n}$, thus we obtain

$$
\nabla_{4 ; n}=o_{\mathbb{P}}(1) .
$$

Therefore from (4.10), (4.12), (4.13), (4.14) and (4.15), we conclude that

$$
\sup _{\mathbf{u} \in[0,1]^{d}}\left|\widehat{\mathbb{A}}_{n}(\mathbf{u})-\mathbb{A}_{n}(\mathbf{u})\right|=o_{\mathbb{P}}(1) .
$$

An application of the triangle inequality shows, in turn, that

$$
\begin{aligned}
\sup _{\mathbf{u} \in[0,1]^{d}}\left|\widehat{\mathbb{A}}_{n}(\mathbf{u})-\frac{1}{\sqrt{n}} \mathscr{K}_{\mathbb{C}}^{*}(\mathbf{u}, n)\right| \leq & \sup _{\mathbf{u} \in[0,1]^{d}}\left|\widehat{\mathbb{A}}_{n}(\mathbf{u})-\mathbb{A}_{n}(\mathbf{u})\right| \\
& +\sup _{\mathbf{u} \in[0,1]^{d}}\left|\mathbb{A}_{n}(\mathbf{u})-\frac{1}{\sqrt{n}} \mathscr{K}_{\mathbb{C}}^{*}(\mathbf{u}, n)\right| .
\end{aligned}
$$

This, when combined with (4.16) and Theorem 2.1, completes the proof of Corollary 3.1.

\section{References}

Adler, R. J. (1990). An introduction to continuity, extrema, and related topics for general Gaussian processes. Institute of Mathematical Statistics Lecture Notes-Monograph Series, 12. Institute of Mathematical Statistics, Hayward, CA. 
Bouzebda, S., El Faouzi, N.-E., and Zari, T. (2011a). On the multivariate two-sample problem using strong approximations of empirical copula processes. Comm. Statist. Theory Methods, 40(8), 14901509.

Bouzebda, S., Keziou, A., and Zari, T. (2011b). K-sample problem using strong approximations of empirical copula processes. Math. Methods Statist., 20(2), 14-29.

Bücher, A. and Dette, H. (2010). A note on bootstrap approximations for the empirical copula process. Statist. Probab. Lett., 80 (23-24), 1925-1932.

Chen, S. X. and Huang, T.-M. (2007). Nonparametric estimation of copula functions for dependence modelling. Canad. J. Statist., 35(2), 265-282.

Cherubini, U., Luciano, E., and Vecchiato, W. (2004). Copula methods in finance. Wiley Finance Series. John Wiley \& Sons Ltd., Chichester.

Chung, K.-L. (1949). An estimate concerning the Kolmogoroff limit distribution. Trans. Amer. Math. Soc., 67, 36-50.

Csörgő, M. (1979). Strong approximations of the Hoeffding, Blum, Kiefer, Rosenblatt multivariate empirical process. J. Multivariate Anal., 9(1), 84-100.

Csörgő, M. and Horváth, L. (1988). A note on strong approximations of multivariate empirical processes. Stochastic Process. Appl., 28(1), 101-109.

Csörgő, M. and Horváth, L. (1993). Weighted approximations in probability and statistics. Wiley Series in Probability and Mathematical Statistics: Probability and Mathematical Statistics. John Wiley \& Sons Ltd., Chichester. With a foreword by David Kendall.

Csörgő, M., Horváth, L., and Szyszkowicz, B. (1997). Integral tests for suprema of Kiefer processes with application. Statist. Decisions, 15(4), 365-377.

Csörgő, M. and Révész, P. (1981). Strong approximations in probability and statistics. Probability and Mathematical Statistics. Academic Press Inc. [Harcourt Brace Jovanovich Publishers], New York.

Csörgó, S. and Hall, P. (1984). The Komlós-Major-Tusnády approximations and their applications. Austral. J. Statist., 26(2), 189-218.

Cui, S. and Sun, Y. (2004). Checking for the gamma frailty distribution under the marginal proportional hazards frailty model. Statist. Sinica, 14(1), 249-267.

DasGupta, A. (2008). Asymptotic theory of statistics and probability. Springer Texts in Statistics. New York, NY: Springer.

Deheuvels, P. (1979). La fonction de dépendance empirique et ses propriétés. Un test non paramétrique d'indépendance. Acad. Roy. Belg. Bull. Cl. Sci. (5), 65(6), 274-292. 
Deheuvels, P. (1980). Nonparametric test of independence. In Nonparametric asymptotic statistics (Proc. Conf., Rouen, 1979) (French), Lecture Notes in Math., Vol. 821, J.P. Raoult, ed., pages 95107. Springer, Berlin.

Deheuvels, P. (1981). Multivariate tests of independence. In Analytical methods in probability theory (Oberwolfach, 1980), volume 861 of Lecture Notes in Math., pages 42-50. Springer, Berlin.

Deheuvels, P. (2009). A multivariate Bahadur-Kiefer representation for the empirical copula process. Zap. Nauchn. Sem. S.-Peterburg. Otdel. Mat. Inst. Steklov. (POMI), 364(Veroyatnost i Statistika. 14.2), 120-147, 237.

Fermanian, J.-D., Radulović, D., and Wegkamp, M. (2004). Weak convergence of empirical copula processes. Bernoulli, 10(5), 847-860.

Frees, E. W. and Valdez, E. A. (1998). Understanding relationships using copulas. N. Am. Actuar. J., 2(1), 1-25.

Komlós, J., Major, P., and Tusnády, G. (1975). An approximation of partial sums of independent RV's and the sample DF. I. Z. Wahrscheinlichkeitstheorie und Verw. Gebiete, 32, 111-131.

Gaenssler, P. and Stute, W. (1987). Seminar on empirical processes, volume 9 of DMV Seminar. Birkhäuser Verlag, Basel.

Joe, H. (1997). Multivariate models and dependence concepts, volume 73 of Monographs on Statistics and Applied Probability. Chapman \& Hall, London.

McNeil, A. J., Frey, R., and Embrechts, P. (2005). Quantitative risk management. Princeton Series in Finance. Princeton University Press, Princeton, NJ. Concepts, techniques and tools.

Moore, D. S. and Spruill, M. C. (1975). Unified large-sample theory of general chi-squared statistics for tests of fit. Ann. Statist., 3, 599-616.

Nelsen, R. B. (2006). An introduction to copulas. Springer Series in Statistics. Springer, New York, second edition.

Omelka, M., Gijbels, I., and Veraverbeke, N. (2009). Improved kernel estimation of copulas: weak convergence and goodness-of-fit testing. Ann. Statist., 37(5B), 3023-3058.

Philipp, W. and Pinzur, L. (1980). Almost sure approximation theorems for the multivariate empirical process. Z. Wahrsch. Verw. Gebiete, 54(1), 1-13.

Piterbarg, V. I. (1996). Asymptotic methods in the theory of Gaussian processes and fields, volume 148 of Translations of Mathematical Monographs. American Mathematical Society, Providence, RI. Translated from the Russian by V. V. Piterbarg, Revised by the author.

Rémillard, B. and Scaillet, O. (2009). Testing for equality between two copulas. J. Multivariate Anal., 100(3), 377-386. 
Rémillard, B. Goodness-of-Fit Tests for Copulas of Multivariate Time Series (December 22, 2010). Available at SSRN: http://ssrn.com/abstract $=1729982$

Rüschendorf, L. (1974). On the empirical process of multivariate, dependent random variables. $J$. Multivariate Anal., 4, 469-478.

Rüschendorf, L. (1976). Asymptotic distributions of multivariate rank order statistics. Ann. Statist., 4(5), 912-923.

Rüschendorf, L. (2009). On the distributional transform, Sklar's theorem, and the empirical copula process. J. Statist. Plann. Inference, 139(11), 3921-3927.

Ruymgaart, F. (1973). Asymptotic Theory for Rank Tests for Independence, MC Tract 43. Ph.D. thesis, Amsterdam: Mathematisch Institut.

Ruymgaart, F. H., Shorack, G. R., and van Zwet, W. R. (1972). Asymptotic normality of nonparametric tests for independence. Ann. Math. Statist., 43, 1122-1135.

Scaillet, O. (2005). A Kolmogorov-Smirnov type test for positive quadrant dependence. Canad. J. Statist., 33(3), 415-427.

Segers, J. (2010). Weak convergence of empirical copula processes under nonrestrictive smoothness assumptions. ArXiv e-prints.

Shorack, G. R. and Wellner, J. A. (1986). Empirical processes with applications to statistics. Wiley Series in Probability and Mathematical Statistics: Probability and Mathematical Statistics. John Wiley \& Sons Inc., New York.

Sklar, A. (1959). Fonctions de répartition à $n$ dimensions et leurs marges. Publ. Inst. Statist. Univ. Paris, 8, 229-231.

Sklar, A. (1973). Random variables, joint distribution functions, and copulas. Kybernetika (Prague), 9 , $449-460$.

Stute, W. (1982). The oscillation behavior of empirical processes. Ann. Probab., 10, 86-107.

Stute, W. (1984). The oscillation behavior of empirical processes: The multivariate case. Ann. Probab., 12, 361-379.

Schweizer, B. (1991). Thirty years of copulas. In Advances in probability distributions with given marginals (Rome, 1990), volume 67 of Math. Appl., Vol. 67, G. Dall'Aglio, S. Kotz, and G. Salinetti, eds., pages 13-50. Kluwer Acad. Publ., Dordrecht.

Tsukahara, H. (2000). Empirical copulas and some applications. Research Report 27, The Institute for Economic Studies, Seijo University.

Tsukahara, H. (2005). Semiparametric estimation in copula models. Canad. J. Statist., 33(3), 357-375. 
van der Vaart, A. W. and Wellner, J. A. (1996). Weak convergence and empirical processes. Springer Series in Statistics. Springer-Verlag, New York. With applications to statistics.

Wichura, M. J. (1973). Some Strassen-type laws of the iterated logarithm for multiparameter stochastic processes with independent increments. Ann. Probability, 1, 272-296. 